\title{
Prediction of chemotherapeutic efficacy using the ratio of neutrophils to lymphocytes in patients with unresectable or recurrent gastric cancer
}

\author{
HUANRAN LIU ${ }^{1 *}$, MINGZHI SONG ${ }^{1,2^{*}}$, FENGQI FANG $^{3}$, XUE GAO $^{4}$, ZHEN ZHANG $^{1}$ and SHOUYU WANG ${ }^{1}$ \\ ${ }^{1}$ Department of Surgery, First Affiliated Hospital of Dalian Medical University, Dalian, Liaoning 116011; \\ ${ }^{2}$ Department of Surgery, Third Affiliated Hospital of Dalian Medical University, Dalian, Liaoning 116200; \\ Departments of ${ }^{3}$ Phymatology and ${ }^{4}$ Diagnostic Pathology, First Affiliated Hospital of Dalian Medical University, \\ Dalian, Liaoning 116011, P.R. China
}

Received August 23, 2014; Accepted July 9, 2015

DOI: $10.3892 / 01.2015 .3575$

\begin{abstract}
In cancer patients, the balance between neutrophil $(\mathrm{N})$ and lymphocyte (L) cell counts fluctuates with the tumor load. The objective of the present study was to determine the implications of the chemotherapy effect by the fluctuations of N/L ratio in patients with unresectable or recurrent gastric cancer. The study participants were identified from a prospective cohort of patients with unresectable or recurrent gastric cancer $(n=135)$. The median $\mathrm{N} / \mathrm{L}$ ratio was 3.23 (range: $0.76-20.45$ ) prior to chemotherapy (pre-chemo-N/L ratio) and 2.55 (range: 1.17-13.45) following 2-4 weeks from when the chemotherapy was completed (post-chemo-N/L ratio), respectively. The median overall survival was 7.9 months. The results demonstrated that the N/L ratio of the post-chemotherapy was significantly reduced compared with the pre-chemotherapy group $(\mathrm{P}<0.001)$. The survival rate for the pre-chemo-N/L ratio $\geq 4.0$ group was significantly reduced compared to the $\mathrm{N} / \mathrm{L}<4.0$ group $(\mathrm{P}=0.01)$. The difference of the pre-chemo-N/L ratio subtracted from the post-chemo-N/L ratio can inflect the chemotherapy effect, respectively. These results indicate that the N/L ratio may be used to predict the potential chemotherapy efficacy in unresectable or recurrent gastric cancer.
\end{abstract}

Correspondence to: Dr Zhen Zhang or Dr Shouyu Wang, Department of Surgery, First Affiliated Hospital of Dalian Medical University, 222 Zhongshan Road, Dalian, Liaoning 116011, P.R. China

E-mail: doctorzz@126.com

E-mail:dmuwsy@sina.com

*Contributed equally

Key words: chemotherapy, unresectable gastric cancer, recurrent, neutrophil/lymphocyte ratio

\section{Introduction}

A number of previous studies have indicated that certain immune cells are essential for the progression of tumors and may serve as good indicators of prognosis (1-5). Patients with lymphocyte infiltration around the tumor, for example, may have a good prognosis due to efficient natural killer cells, or because the lymphocytes are involved in lymphokine-activated tumor cell killing (6-8). However, several lines of evidence indicate that a high density of neutrophils may actually promote tumor growth and metastasis $(9,10)$ and/or suppress lymphocyte activity, leading to counteraction of the antitumor immune response (11). In previous clinical studies, tumor-bearing patients who had granulocytosis were treated by selective granulocytapheresis (a procedure which produces controlled depletion of granulocytes), which resulted in prolonged survival rates $(12,13)$. The neutrophil/lymphocyte $(\mathrm{N} / \mathrm{L})$ ratio, represents the relative number of each of these two major leukocyte populations and indicates fluctuations in their numbers and their likely impact on the progression or prognosis of cancer. A number of studies on gastrointestinal cancer have supported this theory. We have previously reported that the N/L ratio was significantly associated with increased tumor stage and overall survival of patients with gastric and rectal cancer $(14,15)$. One theory is that the high $\mathrm{N} / \mathrm{L}$ ratio reflects the poor status of the immune system against cancer and/or the progression of cancer, but no previous studies have demonstrated that the $\mathrm{N} / \mathrm{L}$ ratio may serve as an independent indicator of chemotherapy effect for patients with unresectable or recurrent gastric cancer. Considerable efforts have been made to identify the parameters that predict the effectiveness of chemotherapy, including computerised tomography (CT) scan and the identification of tumor markers. By contrast, the $\mathrm{N} / \mathrm{L}$ ratio, which is measured relatively easily, may be a valuable index (indicator of chemotherapy efficacy) in the clinical setting for determining patients that may benefit from chemotherapy. Based on these concepts, the present study evaluated whether the baseline $\mathrm{N} / \mathrm{L}$ ratio is a predictor of the effectiveness of chemotherapy in the treatment of unresectable or recurrent gastric cancer. 


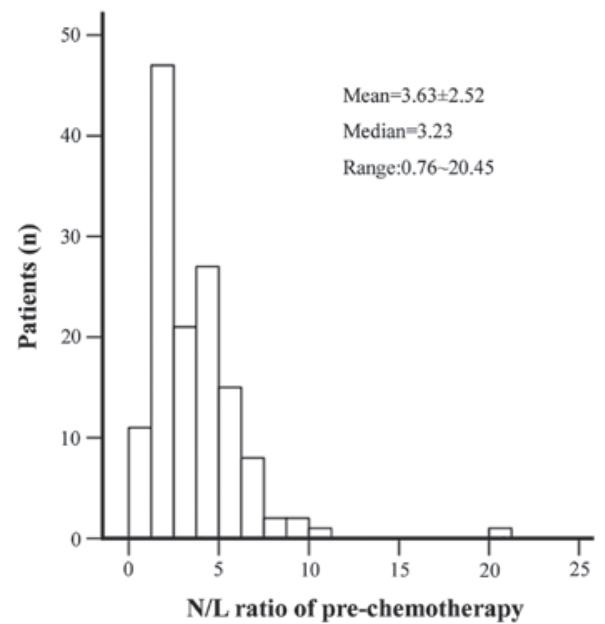

Figure 1. Histogram of the baseline N/L ratio in the peripheral blood of 135 patients with stage IV gastric cancer prior to chemotherapy.

\section{Patients and methods}

A total of 135 patients were selected with unresectable or recurrent gastric cancer who had not previously received adjuvant chemotherapy 1 month prior to randomization (79 males and 56 females; average age, $61.05 \pm 12.05$ years; range, 33-82 years), who were diagnosed using gastroscope biopsy. A total of 56 cases were classified by surgery (aborted the surgery prior to laparotomy or underwent bypass surgery), 70 cases were classified by $\mathrm{CT}$ or magnetic resonance imaging, 9 cases were classified by alternative methods. All the patients received chemotherapy for a 2-6 month course, and patients who objected to bone marrow suppression and/or inflammation were excluded. The N/L ratio was calculated prior to chemotherapy (pre-chemo-N/L ratio) and 2-4 weeks from after the chemotherapy was finished (post-chemo-chemo-N/L ratio). The overall survival was calculated from the date of diagnosis until the date of mortality or the last follow-up time period.

All the data, including gender, age, stage of disease and pathological factors were obtained from the clinical and pathological records and included in the univariate analyses. Significant differences were determined by a non-parametric test. Univariate influence of a given parameter on survival was assessed with log-rank test. $\mathrm{P}<0.05$ was considered to indicate a statistically significant difference. All statistical analyses were performed using SPSS software, version 16 (SPSS, Inc., Chicago, IL, USA).

\section{Results}

The distribution of the baseline pre-chemo-N/L ratio in the 135 patients and the post-chemo-N/L ratio were examined. The median pre-chemo-N/L ratio was 3.23 , the mean was $3.63 \pm 2.52$ (range: $0.76-20.45$ ), with the 25 th and 75 th percentiles 1.85 and 4.55 , respectively (Fig. 1). The post-chemo-N/L ratio was 2.55 , the average was $3.27 \pm 1.83$ (range: 1.17 13.45), with the 25th and 75th percentiles 2.19 and 4.28, respectively (Fig. 2). The N/L ratio of the post-chemotherapy group was significantly reduced compared with the ratio

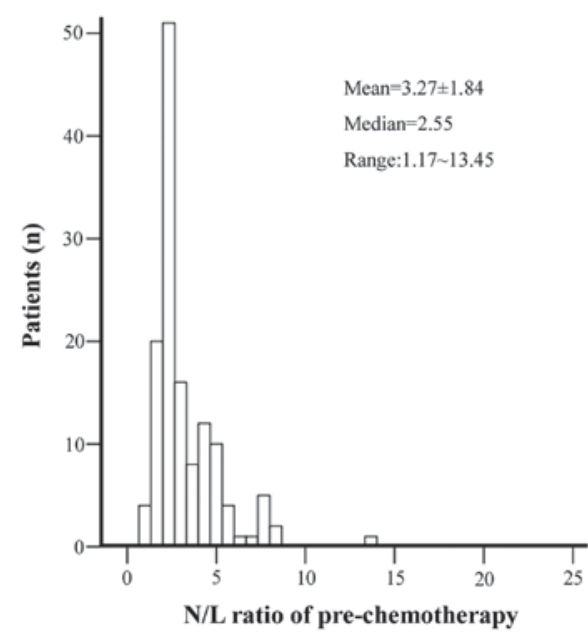

Figure 2. Histogram of the baseline N/L ratio in the peripheral blood of 135 patients with stage IV gastric cancer following chemotherapy.

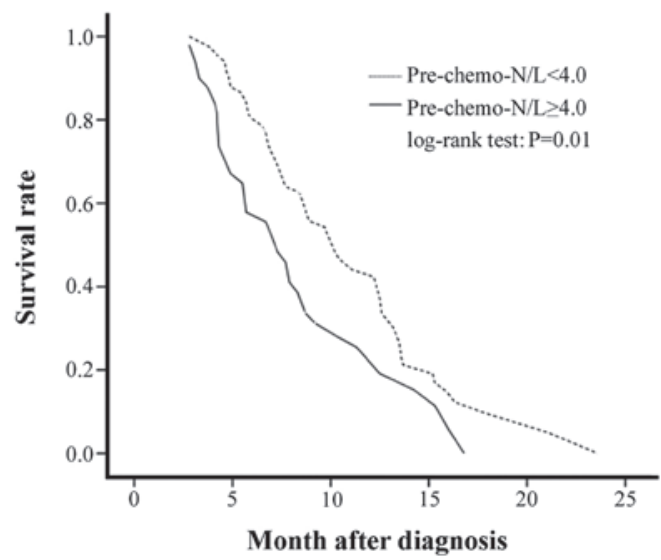

Figure 3. Survival curves based on the pre-chemo-N/L ratio of patients with stage IV gastric cancer. The survival time of the high $\mathrm{N} / \mathrm{L}$ ratio group is reduced compared with the low $\mathrm{N} / \mathrm{L}$ ratio group $(\mathrm{P}=0.01)$.

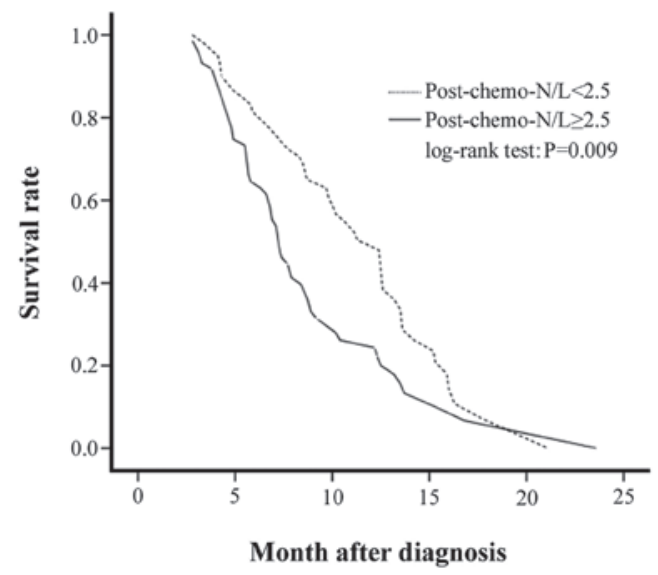

Figure 4. Survival curves based on the post-chemo-N/L ratio of patients with stage IV gastric cancer. The survival time of the high $\mathrm{N} / \mathrm{L}$ ratio group is reduced compared with the low $\mathrm{N} / \mathrm{L}$ ratio group $(\mathrm{P}=0.009)$.

of the pre-chemotherapy group $(\mathrm{P}<0.001)$, as assessed by Pearson's correlation. The median overall survival rate was 


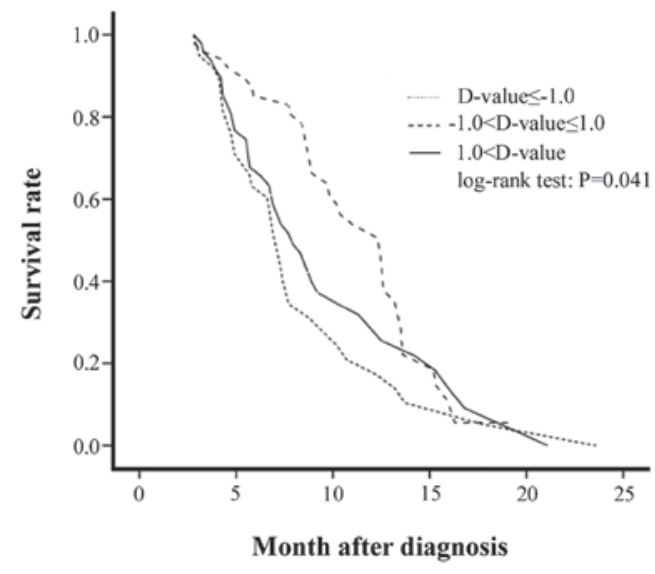

Figure 5. Survival curves based on the D-value (pre-chemo-N/L ratio - postchemo-N/L ratio) in all patients. The median survival rate in patients with $-1.0<\mathrm{D}$-value $\leq 1.0$ (9.8 months) was significantly increased compared with those outside the range (6.85 months in $\mathrm{D} \leq-1.0$ group and 7.2 months in $\mathrm{D}>1.0 ; \mathrm{P}=0.041$.

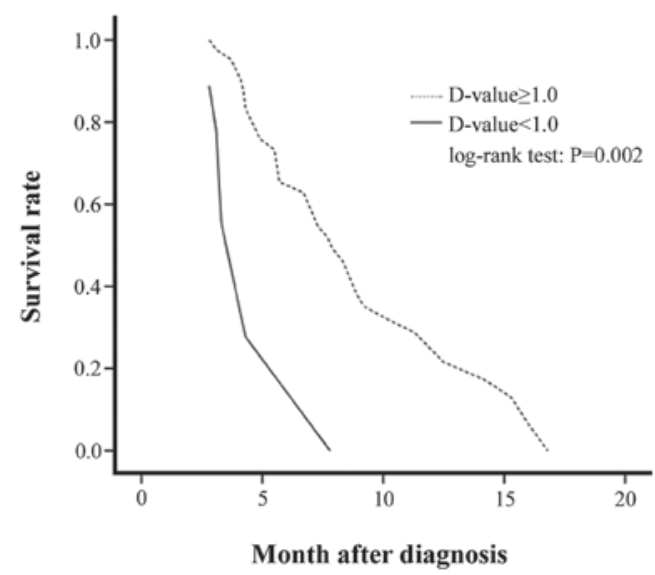

Figure 6. Survival curves based on the D-value (pre-chemo-N/L - postchemo-N/L ratio) in patients with a pre-chemo- $\mathrm{N} / \mathrm{L}$ ratio $\geq 4.0$. The survival rate of the $\mathrm{D}<1.0$ group was reduced compared with the $\mathrm{D} \geq 1.0$ group $(\mathrm{P}=0.002)$

7.9 months, which is longer compared with those patients not treated with adjuvant chemotherapy. The N/L ratio of normal controls is usually $<2.0$ since the proportions of neutrophils and lymphocytes in WBCs are $~ 50-60$ and $30-40 \%$, respectively (16). Thus, compared with normal controls, the $\mathrm{N} / \mathrm{L}$ ratio of enrolled patients was significantly increased. The median overall survival of 8.75 months in the pre-chemotherapy group with an $\mathrm{N} / \mathrm{L}<4.0$, was significantly increased compared with the 6.7 months for the N/L $\geq 4.0$ group ( $\mathrm{P}=0.01$; Fig. 3 ). The median overall survival was 9.7 months in the post-chemo- $\mathrm{N} / \mathrm{L}<2.5$ group, which was significantly increased compared with the median survival rate of 7.0 months in the $\mathrm{N} / \mathrm{L} \geq 2.5$ group $(\mathrm{P}=0.009)$. In order to assess the efficacy of the chemotherapy, the D-value was determined by subtracting the pre-chemo-N/L ratio from the post-chemo-N/L ratio (Fig. 5), the median survival rate for patients with a $\mathrm{D}$-value between -1.0 and 1.0 was significantly increased compared with the patients with $\mathrm{D}$-values outside this range (9.8 months, $\mathrm{P}=0.041)$.

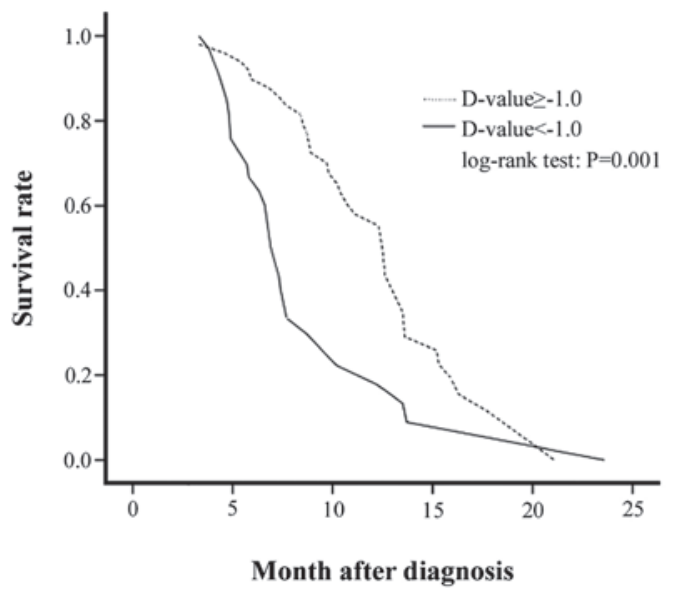

Figure 7. Survival curves based on the D-value (pre-chemo-N/L ratio - postchemo-N/L ratio) in patients with a pre-chemo-N/L ratio $<4.0$. The survival rate of the $\mathrm{D}<-1.0$ group iwas reduced compared with the $\mathrm{D} \geq-1.0$ group $(\mathrm{P}=0.034)$.

The D-value was also determined by subtracting the pre-chemo-N/L ratio from the post-chemo-N/L ratio for the patients who had an N/L ratio $\geq 4.0$ pre-chemotherapy (Fig. 6). The results indicated that the median survival rate of 7.2 months in patients with a $D$-value $\geq 1$ was significantly increased compared with the median survival rate of 3.8 months in patients with a $\mathrm{D}$-value $<1(\mathrm{P}=0.002)$. For the patients who had an $\mathrm{N} / \mathrm{L}$ ratio $<4.0$ pre-chemotherapy, the results indicated that the median survival rate was 10.9 months in patients with a $\mathrm{D}$-value $<1$, which was significantly increased compared with the 6.9 months for patients with a $\mathrm{D}$-value $\geq 1 \quad(\mathrm{P}=0.001)$.

\section{Discussion}

A number of previous studies have indicated that a high neutrophil count is correlated with poor prognosis in patients with various types of cancer, including that of the breast, head and neck and sarcoma. However, previous findings concerning advanced cancer types have also demonstrated that a high count of peripheral neutrophils is an independent prognostic factor for survival rates (17-22). The prognostic role of the N/L ratio has also previously been investigated (15).

The N/L ratio is a good indicator for the evaluation of the condition of a tumor-bearing host $(16,23)$. In humans, the number of circulating neutrophils $(\mathrm{N})$ are increased in the daytime (daytime rhythm), while circulating $\mathrm{T}$ cells, B cells, alphabet $\mathrm{T}$ cells and $\mathrm{CD} 4^{+}$lymphocytes are increased at night (24). Fluctuations in the number of neutrophils are not always in concordance with those of lymphocytes. The N/L ratio as a relative value may correctly reflect fluctuations between neutrophils and lymphocytes and the antitumor efficiency of the host immune mechanism more accurately than the number of circulating neutrophils alone. In the clinic, the efficacy of an antibiotic by the neutrophil count may be predicted, therefore the N/L ratio from a blood test may also potentially be used as a predictor of chemotherapeutic efficacy.

In the present study, the baseline $\mathrm{N} / \mathrm{L}$ ratio in the peripheral blood of patients with stage IV gastric cancer was significantly increased compared with the patients who initially refued 
chemotherapy (median=3.23). Following chemotherapy, the baseline $\mathrm{N} / \mathrm{L}$ ratio was significantly reduced (median $=2.55$ ). It may be that a number of patients benefit simply from the chemotherapy, thus what type of situation may aid in the prediction of the effectiveness of chemotherapy remains to be determined. In the subsequent analysis, an N/L ratio of 4.0 was applied as the cut-off value for patients prior to chemotherapy. The patients were then divided into two groups: The patients with an N/L ratio $\geq 4.0$ had a significantly reduced survival rate compared with those with an N/L ratio $<4.0$, which indicates the reduced prognosis of patients with an increased N/L ratio. In addition, the same results are observed in the post-chemotherapy group when the $\mathrm{N} / \mathrm{L}$ ratio is cut off at 2.5 . The $\mathrm{D}$-value was measured by subtracting the pre-chemo-N/L ratio from the post-chemo-N/L ratio in the high $\mathrm{N} / \mathrm{L}$ ratio group $(\geq 4.0)$; the patients with a D-value $\geq 1$ had a longer survival rate compared with those with a $\mathrm{D}$-value $<1$, which indicates that the former patients benefited from chemotherapy in contrast to the latter group. In the low $\mathrm{N} / \mathrm{L}$ ratio group $(<4.0)$, the patients in $\mathrm{D}$-value $<-1$ group (pre-chemo-N/L ratio subtracted the post-chemo-N/L ratio) had a shorter survival rate compared with those patients with a D-value $\geq-1$, which indicates the former did not benefit from chemotherapy in the same way as the latter. However, in all the patients, the survival rates of the patients in the $-1.0<\mathrm{D}$-value $\leq 1.0$ group was significantly increased compared to the patients with $\mathrm{D}$-values outside of this range $(\mathrm{P}=0.041)$, which indicates that if the immune system of the host is in good functioning condition this results in an improved prognosis. According to the above observations, fluctuations in the $\mathrm{N} / \mathrm{L}$ ratio may allow one to predict the efficacy of chemotherapy.

If a treatment is effective against gastric cancer, the tumor load is reduced following chemotherapy. In a previous study, a positive linear correlation was observed between tumor weight and the N/L ratio within an animal model (14), and an increased $\mathrm{N} / \mathrm{L}$ ratio was associated with poor prognosis because elevated levels of granulocytes promote tumor growth by antagonizing tumor-suppressing lymphocytes. Those results indicate that opposing fluctuations in the neutrophil and lymphocyte counts may be associated with tumor progression.

One possible explanation for this association is that the increased $\mathrm{N} /$ L ratio may reflect an enhanced neutrophil response that promotes tumor growth. Infiltration by lymphocytes has been reported to indicate the generation of an effective antitumor cell immune response (25), and increased lymphocytic infiltration correlates with an improved prognosis (25-27). However, previous findings have shown that tumor-infiltrating granulocytes may promote tumor progression (28), and the involvement of neutrophils in tumor progression has been investigated. In vitro and in vivo experiments have demonstrated that an array of cytokines or other molecules produced by neutrophils contribute to a growth-stimulating microenvironment for cancer cells. Notably, in a number of tumor-transplanted animal models, neutrophils stimulated tumor angiogenesis by increasing proangiogenic factors, including vascular endothelial growth factor (29), interleukin-8 (30), matrix metalloproteinase (31) and elastase (32). Therefore, a high density of circulating neutrophils may exert unfavorable effects on the tumor-bearing host, leading to a negative correlation between neutrophil density and patient survival.
Another possible explanation pertaining to the association of high $\mathrm{N} / \mathrm{L}$ values and poor prognosis is that neutrophils may inhibit the immune system. In support of this theory, neutrophils suppress the cytolytic activity of lymphocytes, natural killer cells and activated $\mathrm{T}$ cells when neutrophils and lymphocytes from normal healthy donors are cultured together, and the degree of suppression is proportional to the number of neutrophils added $(11,33)$. Similarly, an increased $\mathrm{N} / \mathrm{L}$ ratio has been demonstrated to be associated with low immunocompetence in cancer patients $(16,34)$.

In summary, the results of the present study indicate that the peripheral blood $\mathrm{N} / \mathrm{L}$ ratio is an independent indicator of the chemotherapeutic effect on unresectable or recurrent gastric cancer. The accuracy of risk stratification in patients with gastric cancer may lead to more appropriate clinical management in these patients. Therefore, the $\mathrm{N} / \mathrm{L}$ ratio, which is measured relatively easily and at low cost, may be utilized as a potential biomarker of outcome in the clinic. However, further analyses of the prognostic implications of the $\mathrm{N} / \mathrm{L}$ ratio in other types of malignancies are required.

\section{References}

1. Zhang J, Gong F, Li L, Zhao M and Song J: Diabetes mellitus and the neutrophil to lymphocyte ratio predict overall survival in non-viral hepatocellular carcinoma treated with transarterial chemoembolization. Oncol Lett 7: 1704-1710, 2014.

2. Ozdemir Y, Akin ML, Sucullu I, Balta AZ and Yucel E: Pretreatment neutrophil/lymphocyte ratio as a prognostic aid in colorectal cancer. Asian Pac J Cancer Prev 15: 2647-2650, 2014.

3. McNamara MG, Templeton AJ, Maganti M, Walter T, Horgan AM, McKeever L, Min T, Amir E and Knox JJ: Neutrophil/lymphocyte ratio as a prognostic factor in biliary tract cancer. Eur J Cancer 50: 1581-1589, 2014.

4. Williams KA, Labidi-Galy SI, Terry KL, Vitonis AF, Welch WR, Goodman A and Cramer DW: Prognostic significance and predictors of the neutrophil-to-lymphocyte ratio in ovarian cancer. Gynecol Oncol 132: 542-550, 2014.

5. Helal TE, Ibrahim EA and Alloub AI: Immunohistochemical analysis of tumor-infiltrating lymphocytes in breast carcinoma: Relation to prognostic variables. Indian J Pathol Microbiol 56: 89-93, 2013.

6. Allard MA, Bachet JB, Beauchet A, Julie C, Malafosse R, Penna C, Nordlinger B and Emile JF: Linear quantification of lymphoid infiltration of the tumor margin: A reproducible method, developed with colorectal cancer tissues, for assessing a highly variable prognostic factor. Diagn Pathol 7: 156, 2012.

7. Slootweg PJ, de Pagter M, de Weger RA and de Wilde PC: Lymphocytes at tumor margins in patients with head and neck cancer. Relationship with tumor size, human lymphocyte antigen molecules, and metastasis. Int J Oral Maxillofac Surg 23: 286-289, 1994

8. Koshy S, Wu D, Hu X, Tajhya RB, Huq R, Khan FS Pennington MW, Wulff H, Yotnda $\mathrm{P}$ and Beeton C: Blocking KCa3.1 channels increases tumor cell killing by a subpopulation of human natural killer lymphocytes. PLoS One 8: e76740, 2013.

9. Coussens LM and Werb Z: Inflammation and cancer. Nature 420: 860-867, 2002.

10. Tabbekh M, Mokrani-Hammani M, Bismuth G and Mami-Chouaib F: T-cell modulatory properties of CD5 and its role in antitumor immune responses. Oncoimmunology 2: e22841, 2013.

11. el-Hag A and Clark RA: Immunosuppression by activated human neutrophils. Dependence on the myeloperoxidase system. J Immunol 139: 2406-2413, 1987.

12. Tabuchi $T$, Ubukata $\mathrm{H}$, Sato $\mathrm{S}$, Nakata $\approx$ I, Goto $\mathrm{Y}$, Watanabe Y, Hashimoto T, Mizuta T, Adachi M and Soma T: Granulocytapheresis as a possible cancer treatment. Anticancer Res 15: 985-990, 1995.

13. Tabuchi T, Ubukata H, Saniabadi AR and Soma T: Granulocyte apheresis as a possible new approach in cancer therapy: A pilot study involving two cases. Cancer Detect Prev 23: 417-421, 1999. 
14. Liu H, Tabuchi T, Takemura A, Kasuga T, Motohashi G, Hiraishi K, Katano M, Nakada I, Ubukata $\mathrm{H}$ and Tabuchi T: The granulocyte/lymphocyte ratio as an independent predictor of tumour growth, metastasis and progression: Its clinical applications. Mol Med Rep 1: 699-704, 2008.

15. Liu H, Liu G, Bao Q, et al: The baseline ratio of neutrophils to lymphocytes is associated with patient prognosis in rectal carcinoma. J Gastrointest Cancer 41: 116-120, 2010.

16. Satomi A, Murakami S, Ishida K, Mastuki M, Hashimoto T and Sonoda M: Significance of increased neutrophils in patients with advanced colorectal cancer. Acta Oncol 34: 69-73, 1995.

17. Donskov $\mathrm{F}$ and von der Maase $\mathrm{H}$ : Impact of immune parameters on long-term survival in metastatic renal cell carcinoma. J Clin Oncol 24: 1997-2005, 2006.

18. Schmidt H, Suciu S, Punt CJ, et al; American Joint Committee on Cancer Stage IV Melanoma; EORTC 18951: Pretreatment levels of peripheral neutrophils and leukocytes as independent predictors of overall survival in patients with American Joint Committee on Cancer Stage IV Melanoma: Results of the EORTC 18951 Biochemotherapy Trial. J Clin Oncol 25: 1562-1569, 2007

19. Négrier S, Escudier B, Gomez F, Douillard JY, Ravaud A Chevreau C, Buclon M, Pérol D and Lasset C: Prognostic factors of survival and rapid progression in 782 patients with metastatic renal carcinomas treated by cytokines: A report from the Groupe Français d'Immunothérapie. Ann Oncol 13: 1460-1468, 2002.

20. Atzpodien J, Royston P, Wandert T and Reitz M; DGCIN German Cooperative Renal Carcinoma Chemo-Immunotherapy Trials Group: Metastatic renal carcinoma comprehensive prognostic system. Br J Cancer 88: 348-353, 2003.

21. Hashimoto T, Ohno Y, Nakashima J, Gondo T, Ohori M and Tachibana M: Clinical significance of preoperative peripheral blood neutrophil count in patients with non-metastatic upper urinary tract carcinoma. World J Urol 31: 953-958, 2013.

22. Thavaramara T, Phaloprakarn C, Tangjitgamol S and Manusirivithaya S: Role of neutrophil to lymphocyte ratio as a prognostic indicator for epithelial ovarian cancer. J Med Assoc Thai 94: 871-877, 2011.

23. Hirashima M, Higuchi S, Sakamoto K, Nishiyama $T$ and Okada H: The ratio of neutrophils to lymphocytes and the phenotypes of neutrophils in patients with early gastric cancer. J Cancer Res Clin Oncol 124: 329-334, 1998.
24. Suzuki S, Toyabe S, Moroda T, et al: Circadian rhythm of leucocytes and lymphocytes subsets and its possible correlation with the function of the autonomic nervous system. Clin Exp Immunol 110: 500-508, 1997.

25. Rabinowich H, Cohen R, Bruderman I, Steiner Z and Klajman A: Functional analysis of mononuclear cells infiltrating into tumors: Lysis of autologous human tumor cells by cultured infiltrating lymphocytes. Cancer Res 47: 173-177, 1987.

26. Kloor M: Lymphocyte infiltration and prognosis in colorectal cancer. Lancet Oncol 10: 840-841, 2009.

27. Balkwill $\mathrm{F}$ and Mantovani A: Inflammation and cancer: Back to Virchow? Lancet 357: 539-545, 2001.

28. Liu H, Ubukata H, Tabuchi T, et al: It is possible that tumour-infiltrating granulocytes promote tumour progression. Oncol Rep 22: 29-33, 2009.

29. Tan KW, Chong SZ, Wong FH, Evrard M, Tan SM, Keeble J, Kemeny DM, Ng LG, Abastado JP and Angeli V: Neutrophils contribute to inflammatory lymphangiogenesis by increasing VEGF-A bioavailability and secreting VEGF-D. Blood 122 : 3666-3677, 2013

30. Chen L, Li Q, Zhou XD, Shi Y, Yang L, Xu SL, Chen C, Cui YH, Zhang X and Bian XW: Increased pro-angiogenic factors, infiltrating neutrophils and CD163(+) macrophages in bronchoalveolar lavage fluid from lung cancer patients. Int Immunopharmacol 20: 74-80, 2014.

31. Geraghty P, Rogan MP, Greene CM, Boxio RM, Poiriert T, O'Mahony M, Belaaouaj A, O'Neill SJ, Taggart CC and McElvaney NG: Neutrophil elastase up-regulates cathepsin $\mathrm{B}$ and matrix metalloprotease-2 expression. J Immunol 178: 5871-5878, 2007.

32. Scapini P, Nesi L, Morini M, Tanghetti E, Belleri M, Noonan D, Presta M, Albini A and Cassatella MA: Generation of biologically active angiostatin kringle 1-3 by activated human neutrophils. J Immunol 168: 5798-5804, 2002.

33. KayHDandSmithDL:Regulationofhumanlymphocyte-mediated natural killer (NK) cell activity. I. Inhibition in vitro by peripheral blood granulocytes. J Immunol 130: 475-483, 1983.

34. Ietomi K: A study on the role of granulocytes in carcinomabearing hosts-G/L ratio as a new host indicator. Nippon Gan Chiryo Gakkai Shi 25: 662-671, 1990 (In Japanese). 